\title{
Ações da liga acadêmica de terapia nutricional enteral domiciliar no contexto da pandemia
}

\author{
Actions of the academic league of home enteral \\ nutritional therapy in the pandemic context
}

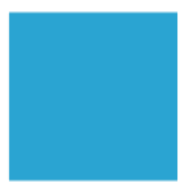

Revista Extensão em Foco
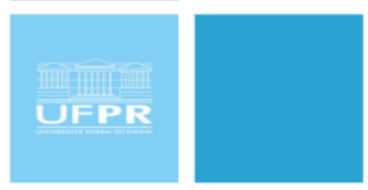

ISSN $2358-7180$

\section{Erika Klingelfus de Almeida Silva ${ }^{1}$, Jennifer Jaqueline de Oliveira ${ }^{2}$, Jade Ferreira Virgilino $^{3}$, Estela Iraci Rabito ${ }^{4}$, Maria Eliana Madalozzo Schieferdecker ${ }^{5}$, Cíbele Pereira Kopruszynski ${ }^{6}$}

\author{
RESUMO
}

\begin{abstract}
A extensão universitária faz parte da formação na universidade, e as ligas acadêmicas estão presente neste contexto, promovendo a inserção de estudantes nas atividades de pesquisa e extensão, no ano de 2020, com a pandemia, essas atividades tiveram de ser adaptadas por conta do isolamento social recomendado pela Organização Mundial de Saúde e foi, justamente, nesse contexto que surgiu a Liga do BHEN (Brazilian Home Enteral Nutrition) vinculada ao Departamento de Nutrição da Universidade Federal do Paraná (UFPR). Objetivo: Relatar a criação e manutenção de uma Liga Acadêmica no contexto da pandemia de COVID-19. Metodologia: Relato de experiência dos membros fundadores da Liga do BHEN envolvendo o período de maio de 2020 a fevereiro de 2021, norteado por registro das atas das reuniões, regimento interno e as normativas de Extensão da UFPR. Resultados: Desde sua criação foram realizados diversos eventos mensais com profissionais convidados, apresentação de resumos em congressos, abertura de processos seletivos, além da, administração das redes sociais a fim de divulgar informações de cunho científico. Considerações finais: Mesmo com a suspensão do calendário acadêmico sendo tão atípico e desafiador, a Liga conseguiu se consolidar e trazer contribuições científicas e sociais para todos os envolvidos.
\end{abstract}

Palavras-chave: Liga acadêmica. Pandemia. Extensão universitária. Nutrição enteral.

\section{ABSTRACT}

University extension is part of training at the university, and the academic league is present in this context with the promotion of the inclusion of students in research and extension activities, in the year 2020, with the pandemic, these activities had to be adapted on account of the social isolation recommended by the World Health Organization and it was precisely in this context that the BHEN League (Brazilian Home Enteral Nutrition) of the Federal University of Paraná (UFPR) emerged. Objectives: Show the creation and maintenance of an Academic League in the context of the COVID-19 pandemic.

1 Discente. Universidade Federal do Paraná (UFPR), Curitiba, Paraná, Brasil. Email:erikaklingelfus@ufpr.br. Orcid: https://orcid.org/0000-0002-4399-4717.

2 Discente. Universidade Federal do Paraná (UFPR), Curitiba, Paraná, Brasil. Email:jenniferoliveira@ufpr.br. Orcid: https://orcid.org/0000-0002-4411-6238.

3 Discente. Universidade Federal do Paraná (UFPR), Curitiba, Paraná, Brasil. E-mail: jade.virgilino@ufpr.br. Orcid: https://orcid.org/0000-0001-7720-6996.

Doutora em Ciências Médicas - FMRP/USP. Universidade Estadual do Paraná (UFPR), Curitiba, Paraná, Brasil. E-mail: rabito@ufpr.br. Orcid: https://orcid.org/0000-0002-3857-9746.

Doutora em Clínica Cirúrgica - UFPR. Universidade Estadual do Paraná (UFPR), Curitiba, Paraná, Brasil. E-mail: meliana@ufpr.br. Orcid: https://orcid.org/0000-0002-9400-3717.

Doutora em Ciências Nutricionais - Unesp/Araraquara. Universidade Federal do Paraná (UFPR), Curitiba, Paraná, Brasil. E-mail: cibele.kopruszynski@ufpr.br . Orcid: https://orcid.org/0000-0003-22877607. 
Methodology: experience report from May 2020 to February 2021, of the founding members, based on the minutes of the meetings, internal regulations and the extension rules of UFPR. Results: Since its creation, several monthly events have been held with professionals, presentation of abstracts at congresses, opening of selection processes, in addition to the administration of social networks in order to disseminate information throughout the community. Conclusions: Even with the academic calendar being so atypical of challenging, the League managed to consolidate itself and bring scientific and social contributions to all those involved.

Keywords: Academic league. Pandemic. University extension. Enteral nutrition.

\section{INTRODUÇÃO}

A extensão universitária é parte integrante da formação dos estudantes, sendo um dos pilares da universidade, onde os alunos são incentivados a compartilhar os conhecimentos adquiridos com os demais setores da sociedade, proporcionando, assim, a oportunidade de interagir diretamente com questões de impacto social, por meio de uma interação dialógica com os usuários, além, de impactar na formação do estudante. A Extensão na Educação Superior Brasileira é a atividade que se integra à matriz curricular e à organização da pesquisa, constituindo-se em processo interdisciplinar, político educacional, cultural, científico, tecnológico, que promove a interação transformadora entre as instituições de ensino superior e os outros setores da sociedade, por meio da produção e da aplicação do conhecimento, em articulação permanente com o ensino e a pesquisa (BRASIL, 2018). A liga acadêmica faz parte das ações previstas neste contexto e caracteriza-se por promover a inserção de estudantes nas atividades assistenciais de pesquisa e extensão (BRASIL, 2018; UFPR, 2019).

Nesse contexto, as ligas apresentam um papel preponderante diante das atividades didáticas que estimulem a criatividade, assim como a iniciativa para a autoaprendizagem e o espírito crítico, preparam o profissional para as constantes transformações e avanços do conhecimento no mundo moderno (BASTOS; TRAJMAN; TEIXEIRA; SELIG; BELO, 2012).

A dinâmica complexa da saúde brasileira exige a formação de profissionais de saúde que possuam, além da ética e capacidade de atuação em vários níveis de atenção, o respeito à cidadania, além do compromisso e a responsabilidade social no processo saúde doença (BRASIL, 2001).

Ao discutir a integração com o social a universidade se mostra aberta para a sociedade permitindo a entrada dos saberes do senso comum no campo da vida acadêmica (VANNUCCHI, 2004). As ações extensionistas geram interferências significativas tanto 
no campo acadêmico quanto na sociedade. A extensão proporciona na academia a geração de novos conhecimentos, a criação de novas modalidades de pesquisa, além da integração entre teoria e prática. Já na sociedade permite uma melhor percepção dos problemas sociais, econômicos e políticos (SANTOS; ROCHA; PASSAGLIO, 2016).

Entretanto, no ano de 2020, diante da situação atípica de crise sanitária que afetou todo o mundo, a principal recomendação da Organização Mundial de Saúde (OMS) foi aderir ao isolamento social, a fim de conter a propagação muito acelerada do Coronavírus. Com isso, a Extensão universitária enfrentou o dilema de como dar sequência às atividades de extensão em meio a uma pandemia.

Deparando-se com esta realidade, a Universidade foi obrigada a desenvolver um plano de enfrentamento, onde os discentes e todos os servidores foram isolados em suas residências, alterando todas as suas atividades de ensino, pesquisa e extensão. No entanto, os problemas para a sociedade só foram aumentando e o desconhecido trouxe novos desafios à academia científica.

Dessa forma a extensão, assim como praticamente todas as atividades acadêmicas nesse novo momento, precisou se reinventar. O que antes era realizado na comunidade, no atual momento de pandemia causado pelo Coronavírus (SARS-CoV-2) passou a ser realizado através das telas de smartphones e computadores, ou seja, no atual contexto de crise na saúde pública faz-se necessário adaptar os processos, de maneira a promover práticas que contribuam para a continuidade e melhor desenvolvimento das ações de extensão, bem como para pensar novas formas de conduzir tais ações (BARBOSA, 2020).

Assim, no final de 2019 foi idealizado por parte de docentes e discentes do Curso de Graduação em Nutrição da Universidade Federal do Paraná um projeto de extensão, denominado "Liga Acadêmica de Terapia Nutricional Enteral Domiciliar" (LATNED), a primeira liga acadêmica em 40 anos do curso de nutrição da UFPR, que busca através do ensino, pesquisa e extensão ampliar o conhecimento acadêmico a fim de favorecer a comunidade usuária de TNED e nutricionistas que atuam em diversos níveis de assistência. Porém seu início, se deu justamente neste contexto pandêmico e de suspensão das atividades acadêmicas presenciais.

Foi instituída com objetivos, que visam contribuir para os usuários e cuidadores, além de estudantes e profissionais que atuam em TNED, destacando: atualizar, aprofundar e difundir conhecimentos e técnicas em terapia nutricional enteral domiciliar, desenvolver atividades assistenciais em terapia nutricional sob supervisão de docentes e 
nutricionistas, estimular e promover o ensino e a pesquisa, servindo-lhes de campo de atividades e desenvolvimento, realizar ações de integração com a comunidade interna e externa, refletindo e discutindo questões de cunho científico, cultural e social.

A liga surgiu como uma ramificação do grupo de pesquisa em Terapia Nutricional Enteral Domiciliar, que passou a ser denominado de "Brazilian Home Enteral Nutrition" - Grupo do BHEN, o qual desenvolve atividades e pesquisas científicas em parceria com diversas instituições públicas e privadas, visando a expansão internacional e buscando parcerias com outros grupos e pesquisadores. O grupo do BHEN também promove discussões periódicas entre profissionais e estudantes de graduação e pós-graduação que atuam ou desejam aprofundar seus conhecimentos sobre terapia nutricional enteral domiciliar (TNED).

Dessa forma a Liga acadêmica do BHEN vem se fortalecendo pela troca de informações e experiências junto aos professores, nutricionistas, alunos de pós-graduação e graduação que participam do grupo de pesquisa, o que vem possibilitando o contato com diferentes pontos de vista, que contribuem fortemente para a construção de uma visão mais crítica e analítica (ROSSIT et. al., 2018). Nesse sentido a TNED é o foco das discussões, sendo contextualizada todas suas interfaces.

A TNE consiste em realizar a alimentação e a nutrição de um indivíduo por via alternativa, ou seja, através de sonda ou ostomia, onde o paciente recebe os nutrientes necessários para a manutenção ou recuperação do estado nutricional (BRASPEN, 2018). Enquanto que a TNED permite que o paciente dê prosseguimento ao seu tratamento nutricional em casa, sendo fundamental que o paciente, ou seu cuidador tenham o conhecimento necessário em relação aos cuidados que envolve o preparo, o armazenamento e a administração da alimentação, até a higienização da sonda e a monitorização contínua desses cuidados (SCHIEFERDECKER; THIEME, 2019).

Nesse contexto, a LATNED pode contribuir com orientações técnicas atualizadas e seguras visando a educação em saúde aos pacientes e cuidadores e no conhecimento dos acadêmicos.

Portanto, o objetivo deste artigo é descrever o processo de criação da LATNED, bem como relatar suas atividades extensionistas em meio a um ano com calendário acadêmico atípico, ocorrido entre maio de 2020 a fevereiro de 2021.

\section{METODOLOGIA}


Trata-se de um relato de experiência expresso por membros fundadores da liga, correspondendo ao período de maio de 2020 a fevereiro de 2021, envolvendo todos os processos e atividades que fizeram parte da criação e desenvolvimento de um projeto de extensão universitária, intitulado "Liga Acadêmica de Terapia Nutricional Enteral Domiciliar".

Como material norteador para o desenvolvimento deste relato foram utilizados registros das atas das reuniões ordinárias e extraordinárias da Liga, bem como o regimento interno da LATNED e as normativas de Extensão da UFPR.

A LATNED foi fundada por 3 docentes do Departamento de Nutrição da UFPR, e por discentes regularmente matriculados e vinculados a três diferentes instituições de ensino superior na cidade de Curitiba/PR.

A agenda da LATNED é constituída por reuniões ordinárias mensais, e reuniões extraordinárias de acordo com a necessidade. A LATNED conta com um cronograma mensal de eventos, podendo ser restrito às ligantes ou aberto à comunidade. Participa também nas reuniões ordinárias e de brainstorm do grupo BHEN a qual a LATNED é vinculada e participa ativamente.

\section{RESULTADOS E DISCUSSÃO}

Em maio de 2020 houve a primeira reunião de apresentação e consolidação da LATNED, tendo a necessidade de desenvolver coletivamente o regimento, o qual foi concebido de acordo com as normativas da UFPR.

Para Silva e Flores (2015), os princípios que norteiam as ações das Ligas estão contidos em estatutos que estabelecem a denominação, os fins e os requisitos de admissão e exclusão dos membros, os direitos e deveres, o modo de constituição e de funcionamento da Liga, as condições para disposições regimentais e dissolução, e a forma de gestão administrativa, sendo tais normativas essenciais para a sua existência. Factível foi o desafio, por parte dos envolvidos na criação e formalização envolvendo o planejamento de todas as ações de modo remoto por aplicativo na web, processo que envolveu também os eventos online, divulgação e emissão de certificados, ações estas a fim de respeitar o isolamento social característico da pandemia.

Por fim, a identidade visual da LATNED foi criada e está demonstrada na figura 1. 
Figura 1- Logo da LATNED

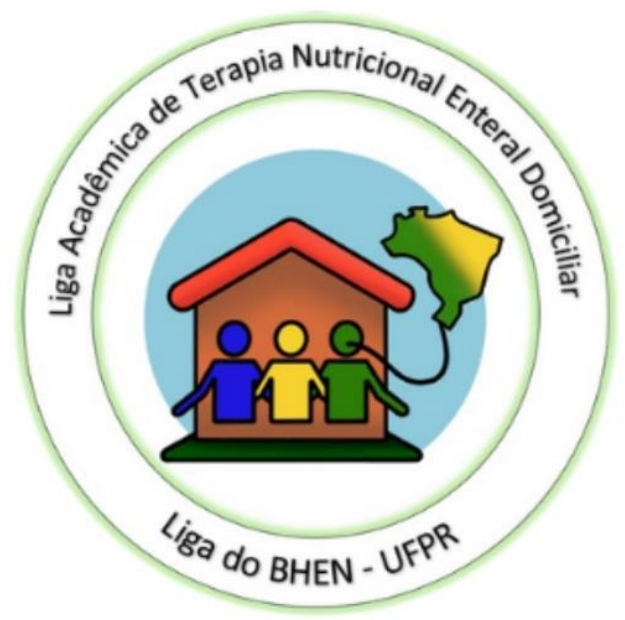

Fonte: LATNED (2020)

Desde sua criação em maio de 2020 até fevereiro de 2021 foram realizadas diversas atividades como a elaboração de eventos mensais com a comunidade acadêmica e profissionais externos. Atuou em conjunto com diversas ligas acadêmicas na organização do "Simpósio Integrado de Cuidados paliativos e Luto" - evento de grande alcance da Liga, com mais de três mil inscrições preenchidas e com grande número assistindo simultaneamente via Youtube, o referido evento foi gravado e já conta com mais de cinco mil visualizações, com a palestra de uma das docentes da LATNED a qual ministrou o tema "Terapia Nutricional Enteral Domiciliar em Cuidados Paliativos". A Liga ainda apresentou diversos trabalhos na forma de resumos no "Congresso brasileiro de saúde pública", além de reuniões mensais para discussão de artigos científicos, e a abertura de dois processos seletivos, um em setembro de 2020, e outro em fevereiro de 2021. A figura 2 apresenta um resumo das atividades da LATNED.

Figura 2 - Linha do tempo da LATNED 


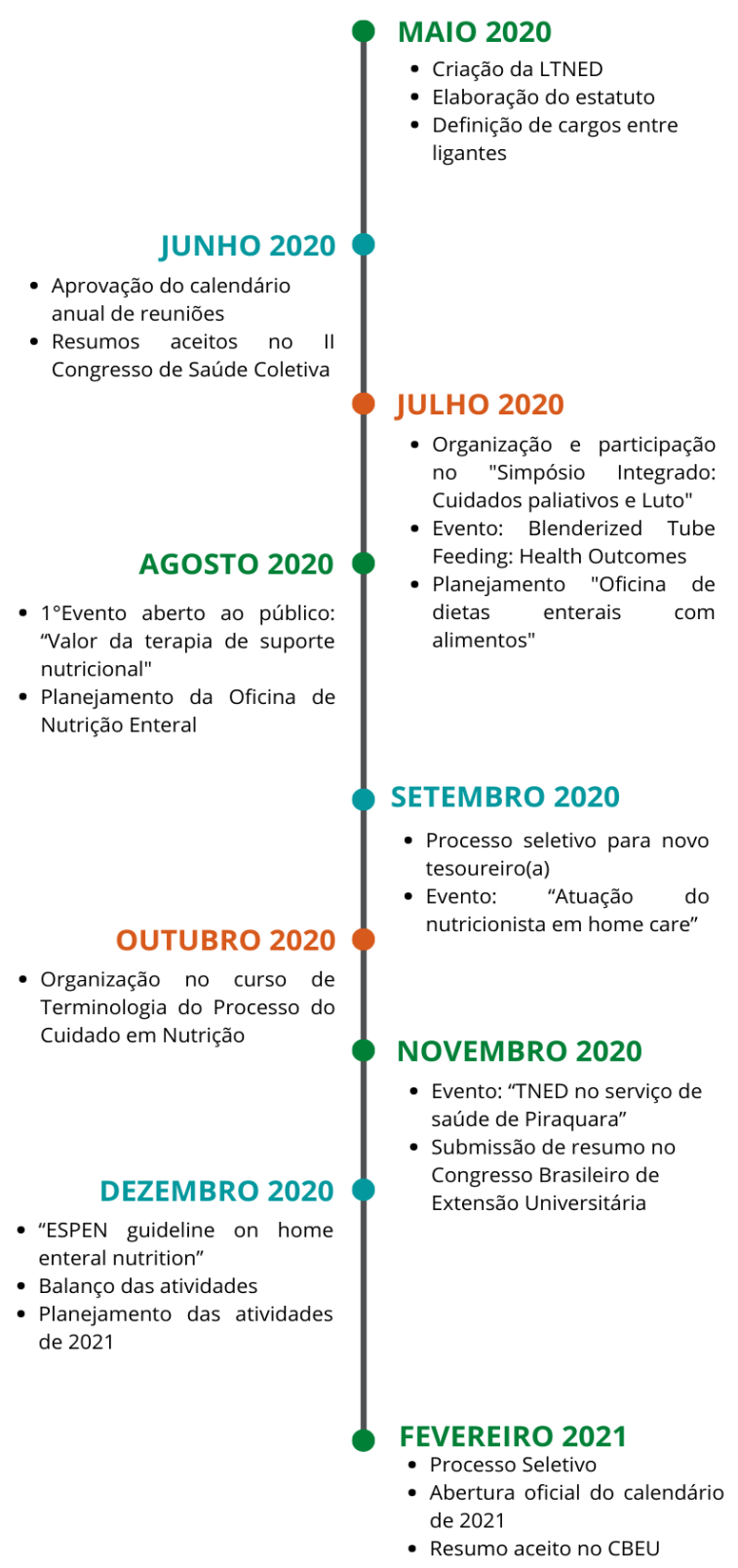

Fonte: As autoras (2021)

Assim, além dos eventos abertos à comunidade acadêmica, as ligantes tiveram a oportunidade de aprofundar o conteúdo referente a Terapia Nutricional Enteral em eventos fechados que visam a qualificação dos membros da liga, contando com a presença das professoras em todas as reuniões e de nutricionistas convidadas que atuam em diferentes níveis de assistência à saúde em Curitiba e região metropolitana.

Os componentes da liga têm a oportunidade em assistir defesas de mestrado referentes ao tema, ter acesso à leituras científicas, estudos de casos clínicos, diretrizes e guidelines. Além do aprofundamento supracitado, a diretoria e coordenação da Liga 
possuem frequência fixa de reuniões remotas a fim de programar os eventos e atividades subsequentes, assim como a atribuição de cada ligante nas atividades planejadas.

Exemplo de oportunidade de aprofundamento foi o Workshop de Terminologia no Processo do Cuidado em Nutrição Clínica, tal evento foi planejado e desenvolvido para nutricionistas e estudantes do último ano da graduação, entretanto as ligantes tiveram a oportunidade de participarem e discutirem os casos clínicos e as atividades propostas.

Nesse contexto, o aprofundamento favorece a diversificação de cenários, proporcionando uma aproximação entre o estudante e as necessidades de saúde da comunidade. Ao mesmo tempo, possibilita um olhar a respeito do trabalho em equipe e a vivência em distintos níveis de complexidade da atenção à saúde proporcionada por profissionais que atuam e discutem coletivamente suas práticas. (BASTOS; TRAJMAN; TEIXEIRA; SELIG; BELO, 2012).

Também há a administração das redes sociais instagram e facebook, os conteúdos neles divulgados são de autoria tanto da liga acadêmica quanto do grupo BHEN e buscam o engajamento social através dos posts, objetivando também a disseminação de informações referentes a TNED a toda a comunidade.

\section{CONCLUSÃO}

Diante deste calendário acadêmico atípico, a LATNED conseguiu se consolidar de forma estruturada e contribuir de diversas formas para o conhecimento dos acadêmicos e da comunidade sobre a terapia nutricional enteral domiciliar, de forma remota, por meio de discussões de artigos, elaboração de simpósio e palestras e pela disseminação de informações técnicas por meio das mídias sociais, além de outras atividades que atingem os objetivos centrais da extensão universitária.

Em suma, a criação e manutenção da liga acadêmica foi desafiadora, porém, gratificante e muito importante no cenário acadêmico durante a pandemia de covid 19, pois trouxe diversas contribuições de cunho científico e social para todos os envolvidos. 


\section{REFERÊNCIAS}

BARBOSA, D. S. Saberes e Práticas da Extensão Universitária na Resposta ao Enfrentamento da COVID-19 no Brasil. Revista Práticas Em Extensão, v. 4, n. 1, p. 5051, 2020.

BASTOS, M.L.S; TRAJMAN, A; TEIXEIRA, E. G; SELIG, L; BELO, M.T.C.T. J. O papel das ligas acadêmicas na formação profissional. Jornal Brasileiro de Pneumologia. 2012;38(6):803-805.

BRASIL. Ministério da Educação. Conselho Nacional de Educação. Câmara de Educação Superior. Resolução no 5 de 7 de novembro de 2001.

BRASIL. Ministério da Educação. Conselho Nacional de Educação. Câmara de Educação Superior. Resolução no 7 de 18 de dezembro de 2018.

BRASPEN - Brazilian Society of Parenteral and Enteral Nutrition. Diretrizes

Brasileira de Terapia Nutricional. Vol. 33 - $1^{\circ}$ suplemento, São Paulo, 2018.

ROSSIT, R.A.S; SANTOS, C.F.J; MEDEIROS, N.M.H; MEDEIROS, L.M.O.R; REGIS, C.G; BATISTA, S.H.S.S. The research group as a learning scenario in/on Interprofessional Education: focus on narratives. Interface (Botucatu). 2018; 22(Supl. 2):1511-23.

SANTOS, J.H.S; ROCHA, B.F; PASSAGLIO, K.T. Extensão Universitária e $7, \mathrm{n}$.

Formação no Ensino Superior. Revista Brasileira de Extensão Universitária. v.

1, p.23-28 jan. - jun. 2016 e-ISSN 2358-0399

SCHIEFERDECKER, M.E.M; THIEME, R.D. Terapia nutricional domiciliar. Rubio, Rio de Janeiro, 2019.

SILVA, S.A.da; FLORES, O. Ligas Acadêmicas no Processo de Formação dos

Estudantes. Revista Brasileira de Educação Médica. Brasília. 39 (3), 410-425, 2015 .

UNIVERSIDADE FEDERAL DO PARANÁ. Conselho de Ensino, Pesquisa e Extensão. Resolução no 57 de dezembro de 2019.

VANNUCCHI, A. A Universidade comunitária: o que é, como se faz. São Paulo: Loyola, 2004.

Recebido em: 25 de Março de 2021.

Aceito em: 14 de Abril de 2021. 